\title{
Treinamento físico aeróbio previne à hipertrofia cardíaca patológica e melhora a função diastólica em ratos Zucker obesos
}

CDD. 20.ed. 616.398

796.073

\author{
Diego Lopes Mendes BARRETTI ${ }^{* / * *}$ \\ Everton Crivoi do CARMO* \\ Kaleizu Teodoro ROSA*** \\ Maria Claudia Costa IRIGOYEN $N^{* * * *}$ \\ Edilamar Menezes de OLIVEIRA*/**
}

*Escola de Educacão Física e Esporte,

Universidade de São Paulo.

**Faculdade de Medicina, Universidade de São Paulo.

***Instituto do Coração, Faculdade de Medicina, Universidade de São Paulo.

\section{Resumo}

A obesidade é uma patologia diretamente relacionada com o desenvolvimento de doenças cardiovasculares. Por outro lado, o treinamento físico aeróbio atenua o desenvolvimento da obesidade e promove benefícios cardíacos em obesos. Dessa forma, nosso objetivo foi investigar se a obesidade altera a função cardíaca e se sua associação com o treinamento físico aeróbio promove melhora na função cardíaca em ratos Zucker obesos. Os ratos Zucker foram divididos da seguinte forma: grupo magro (GM), grupo obeso (G0), grupo magro treinado (GMTR) e grupo obeso treinado (GOTR). 0 protocolo de treinamento aeróbio de natação foi realizado por um período de 10 semanas com cinco sessões semanais de 60 minutos de duração. A frequência cardíaca de repouso, a pressão arterial sistólica, a hipertrofia e função cardíaca foram avaliadas no final do período de treinamento físico. Ambos os grupos treinados apresentaram uma queda de $12 \%$ da frequência cardíaca de repouso, quando comparado com seus respectivos controles. Ainda, nossos resultados demonstraram que o treinamento aeróbio reduziu o aumento da massa cardíaca em 13\% e melhorou a função diastólica na obesidade em $43 \%$. Em conclusão, nossos dados demonstraram que o treinamento físico aeróbio reverteu os prejuizos cardíacos causados pela obesidade.

Unitermos: Obesidade; Cardiopatias; Treinamento físico aeróbio; Ratos Zucker.

\section{Introdução}

Dados publicados pela Organização Mundial da Saúde (OMS) mostram que estilo de vida sedentário e sobrepeso estão entre os maiores problemas de saúde pública mundial, sendo a obesidade uma das doenças com maior expansão em países ocidentais, com aproximadamente 1,7 bilhōes de indivíduos com sobrepeso na população mundial (DEITEL, 2003).

O sobrepeso ocorre devido ao desequilíbrio entre atividade física e o consumo energético da dieta. Assim, estilo de vida sedentário e dieta inadequada levam o indivíduo ao sobrepeso e obesidade, o que aumenta consideravelmente o risco do desenvolvimento de diabetes tipo 2, dislipidemias e doenças cardiovasculares (ABEL, LITWIN \& SWEENEY, 2008).

Dados da literatura mostram uma relação direta entre elevado índice de massa corporal (MC) e aumento do risco de desenvolvimento de insuficiência cardíaca (Poirier, Giles, Bray, Hong, Stern, Pi-Sunyer \& ECKel, 2006). Vários mecanismos têm sido discutidos para explicar essa correlação, incluindo alterações hemodinâmicas com sobrecarga cardíaca e remodelamento ventricular, entretanto estes mecanismos não explicam completamente o desenvolvimento da disfunção cardíaca em indivíduos obesos (Kenchaiah, Evans, Levy, Wilson, Benjamin, LaRSON, KanNel \& VASAN, 2002). Estudos com ratos Zucker obesos demonstram que a disfunção diastólica pode ser ocasionada pelo acúmulo de lipídios e fibrose cardíaca (Toblli, CaO, DeRosa \& Forcada, 2005; Toblui, Cao, Rivas, DeRosa \& DomecQ, 2006). Além disso, essas alteraçōes morfológicas e estruturais são acompanhadas por alteraçôes moleculares no padrão de expressão gênica dos marcadores de hipertrofia cardíaca patológica, como o aparecimento da alfa 
actina esquelética, do fator natriurético atrial (ANF) e da beta miosina de cadeia pesada (BMCP) (GUPTA, 2007). Golfman, Wilson, Sharma, Burgmaier, Young, Guthrie, Van Arsdall, Adrogue, Brown e Taegtmeyer (2005), mostraram aumento da expressão da $\beta \mathrm{MCP}$ sem alterações da $\alpha \mathrm{MCP}$ em ratos Zucker obesos.

Estas alterações moleculares são fatores determinantes da capacidade contrátil do miocárdio, o que pode levar à diminuição na velocidade de encurtamento dos sarcômeros resultando em disfunção cardíaca (IzUMO, NADAL-GinARD \& MAHDAVI, 1988; SWynghedAUW, 2006).

Por outro lado, a disfunção cardíaca causada pela obesidade, pode, em parte, ser revertida com o treinamento físico (TF) aeróbio. OTF aeróbio promove melhora na função diastólica de ratos (BRENnER,

\section{Métodos}

\section{Amostra}

Neste estudo foram utilizados Ratos Zucker obesos (20 semanas, 500-520 g, $\mathrm{n}=11$ ) e ratos Zucker magros (20 semanas, 320-350 g, $\mathrm{n}=10$ ) como controle. Os animais foram adquiridos do Biotério da Escola Paulista de Medicina (UNIFESP) e mantidos no biotério do Laboratório de Bioquímica e Biologia Molecular do Exercício da EEFE-USP com ração e água "ad libitum”, em gaiolas com quatro animais, em sala climatizada (23 $\pm 1^{\circ} \mathrm{C}$ ) e ciclo claro-escuro invertido a cada $12: 12-\mathrm{h}$ durante o experimento.

Os animais foram divididos em quatro grupos: 1) grupo magro (GM); 2) grupo obeso (GO); 3) grupo magro treinado (GMTR); 4) grupo obeso treinado (GOTR). Os grupos GM e GO permaneceram sedentários ao longo do protocolo, enquanto que os grupos GMTR e GOTR realizaram treinamento físico aeróbio de natação. Os ratos foram identificados e pesados semanalmente.

\section{Procedimentos}

Todos os procedimentos cirúrgicos e protocolos foram realizados de acordo com os Princípios Éticos de Experimentação Animal (COBEA, 1991). O projeto de pesquisa foi aprovado pelo Comitê de Ética ( $n^{\circ}$ 1023/07) em Pesquisa da Faculdade de Medicina da Universidade de São Paulo.
APSTEIN \& SAUPE, 2001) e gera um padrão molecular fisiológico, diferente do encontrado na hipertrofia cardíaca patológica, com diminuição da expressão gênica e proteica da $\beta M C P$ e aumento da $\alpha M C P$ (Jin, Yang, Li, Lu, Ryan, OGasawar, Van Peborgh \& PAONI, 2000), o que demonstra o efeito benéfico do TF. Contudo, os efeitos da associação do TF aeróbio com a obesidade nas alteraçôes moleculares de $\alpha$ e $\beta \mathrm{MCP}$, juntamente como os parâmetros funcionais ecocardiográficos são pouco conhecidos.

Dessa forma, uma vez que as alteraçôes na massa e função cardíaca em obesos estão relacionadas com modificações moleculares e o TF pode alterar esses parâmetros trazendo benefícios cardiovasculares, este estudo tem como objetivo investigar se o TF aeróbio de natação pode prover alterações moleculares cardíaca e reverter a disfunção diastólica observada em ratos Zucker obesos.

\section{Treinamento físico aeróbio}

O treinamento de natação foi realizado segundo protocolo adaptado de Medeiros, Oliveira, Gianolla, Casarini, Negrão e Brum (2004), em sistema de natação com água aquecida entre 30-32 ${ }^{\circ} \mathrm{C}$. O treinamento teve duração de 10 semanas, com frequência de cinco vezes por semana. $\mathrm{O}$ tempo e a sobrecarga (peso na cauda do animal) tiveram aumento gradual, sendo que no início do protocolo os animais começaram com 15 minutos e sem carga, chegando até 60 minutos, e $5 \%$ da MC no final da segunda semana.

Este protocolo foi caracterizado como treinamento de baixa intensidade e longa duração, sendo efetivo na promoção de adaptaçóes cardiovasculares e no aumento da capacidade oxidativa muscular (Medeiros et al., 2004).

\section{Avaliação cardiorrespiratória}

\section{Protocolo de exercício físico progressivo até a exaustão}

Antes da realização do teste os animais foram submetidos a um período de adaptação. Conforme descrito abaixo no protocolo do consumo de oxigênio. Após essa etapa, os animais realizam um teste progressivo até a exaustão utilizando protocolo com velocidade inicial de $6 \mathrm{~m} / \mathrm{min}$, sendo 
intensificado a cada três minutos com velocidade de mais $3 \mathrm{~m} / \mathrm{min}$, até chegar o instante que o animal não conseguiu mais manter o padrão de corrida. O teste de esforço físico máximo é utilizado como uma ferramenta para avaliar o desempenho físico após um período de TF aeróbio. Sendo assim, visando testar a eficácia do treinamento, os animais foram submetidos ao teste em esteira rolante até a exaustão. A caracterização da incapacidade de correr dos animais pode ser quantificada por parâmetros obtidos no teste como a distância percorrida, tempo e velocidade máxima de execução do teste (BernsteIn, 2003).

\section{Protocolo do consumo de oxigênio}

Antes da realização da medida do consumo de oxigênio, os animais foram submetidos a um período de adaptação ao exercício, que consistiu na prática de corrida durante 10 minutos, utilizando velocidades variadas, por três dias alternados. A medida do consumo de oxigênio em ratos consiste na utilização de uma caixa metabólica conectada a um analisador de gases capaz de fornecer a concentração de oxigênio no interior da mesma (Brooks \& White, 1978). Nesse estudo, foi utilizada uma caixa metabólica $(9,5$ x 32,5 x 11,5 cm) subdividida, internamente na sua porção superior $(0,9 \mathrm{~cm})$, por uma placa que possui 64 furos ( $0,3 \mathrm{~cm}$ de diâmetro) e que serve como uma câmara de mistura. A essa câmara foi conectado um tubo na forma de "Y" por onde parte da amostra foi retirada por uma bomba (Fanen, mod. Cal) e, outra parte, com menor fluxo, foi retirada pela bomba do analisador de gases (Sable Systems Subsampler Version 3, SS-3). A parte da frente da caixa possui uma abertura de $0,2 \mathrm{~cm}$ da superfície, que permite a entrada de um fluxo de ar unidirecional sugado pelas bombas aspiradoras. Para a realização da medida do consumo de oxigênio durante a realização do exercício físico, a caixa metabólica foi posicionada sobre uma esteira rolante.

\section{Medidas hemodinâmicas}

\section{Avaliação de pressão arterial sistólica}

A pressão arterial sistólica (PAS) foi aferida na última semana do protocolo de treinamento, com um intervalo de 40 horas da sessão de treino que precedeu a medida. $\mathrm{O}$ método utilizado foi pletismografia da artéria caudal (registro indireto da pressão). Os animais foram mantidos sob restrição de movimentos em caixa de acrílico e submetidos a aquecimento moderado, para promover vasodilatação da artéria caudal. O registro da PAS caudal foi realizado através da colocação de um manguito de borracha na região proximal da cauda e ligado ao esfigmomanômetro para insuflar e desinsuflar gradualmente o manguito de 0 a 250/300 $\mathrm{mmHg}$.

Numa porção mais distal da cauda foi acoplado um transdutor pneumático para detecção dos sinais de passagem da onda de pulso de pressão arterial na artéria caudal e registrado no sistema AT/CODAS (DataQ Instruments, Inc., Ohio, USA), com frequência de amostragem de 1000 $\mathrm{Hz}$. A PAS caudal equivale a pressão do manguito, em que o pulso de pressão desaparece ou reaparece, quando a pressão exercida sobre a cauda tornar-se ligeiramente menor que o valor da pressão intraarterial, desobstruindo o fluxo sanguíneo na artéria caudal e permitindo a detecção do pulso de pressão.

A frequência cardíaca (FC) foi calculada a partir do intervalo de tempo de cada pulso de pressão arterial detectado pelo transdutor sem a oclusão da passagem do fluxo sanguíneo na cauda. Cinco medidas da PAS e FC em repouso foram realizadas para cada animal, sendo desprezadas a primeira e a última medida e calculada a média aritmética entre os valores restantes.

\section{Avaliação da função ventricular}

A avaliação da função ventricular foi realizada por ecocardiografia. As medidas ecocardiográficas seguiram as recomendaçóes do Comitê de Padronização do modo M da Sociedade Americana de Ecocardiografia (O’Rourke, Hanrath, Henry, Hugenholz, Pisa, Roelandt \& Tanaka, 1984). É importante salientar que a acurácia e reprodutibilidade do exame ecocardiográfico transtorácico em estimar o tamanho e a função do ventricular em roedores têm sido confirmada em uma série de estudos (SChiller, Shah, CraWford, DeMaria, Devereux, Feigenbaum, Gutgesell, Reichek, Sahn \& Schnittger, 1989). O exame ecocardiográfico transtorácico foi realizado na última semana de protocolo de TF, em todos os grupos. Os exames foram realizados por um único observador e em cada exame foi coletado um total de três medidas para cada variável, sendo calculadas posteriormente as médias dessas medidas. O exame ecocardiográfico foi realizado com os animais anestesiados, por via intraperitoneal, com uma mistura de Xilasina $(0,67 \mathrm{mg} / \mathrm{kg})$ e Ketamine 
$(0,33 \mathrm{mg} / \mathrm{kg})$. O animal anestesiado foi colocado em decúbito lateral, em mesa cirúrgica apropriada. Foi utilizado o equipamento Sonos 5500 (Philips, Andover, Mass) com transdutor de 5 a $12 \mathrm{MHz}$, que permite imagens com 2 ou $3 \mathrm{~cm}$ de profundidade. Para o registro das imagens e da FC foi utilizado o posicionamento de três eletrodos. Desta forma, foram obtidas vistas longitudinais e transversais do para-esternal e vista apical da cavidade 2 e 4 do coração, para a avaliação do fluxo mitral e da velocidade da fração de ejeção do ventrículo esquerdo através do Doppler. As imagens foram armazenadas em fitas de videocassete ou em discos ópticos, para posterior análise.

\section{Função sistólica do ventrículo esquerdo}

O parâmetro foi determinado pela mudança da área fracional, que corresponde à diferença da área diastólica pela área sistólica, dividida pela área diastólica e multiplicada por 100, para representação em porcentagem em três regiōes (basal, média e apical) e pela fração de ejeção utilizando o método de Simpson (Litwin, Katz, Morgan \& Douglas, 1994).

\section{Função diastólica do ventrículo esquerdo}

Este parâmetro foi analisado usando os valores derivados da curva de velocidade de enchimento na diástole, pela fração de ejeção do ventrículo esquerdo realizado pelo Doppler. Outro parâmetro realizado no Doppler para avaliar a função diastólica foi o tempo de relaxamento isovolumétrico (TRIV).

\section{Massa do ventrículo esquerdo}

A massa do ventrículo esquerdo (MVE) foi calculada segundo a orientação da Sociedade Americana de Ecocardiografia, que estima a MVE por meio da utilização da seguinte fórmula matemática $\mathrm{MVE}=\left[(\mathrm{DDVE}+\mathrm{SIP}+\mathrm{PP})^{3}-(\mathrm{DDVE})^{3}\right] \times 1,047$, onde (DDVE) é o diâmetro diastólico do ventrículo esquerdo, (SIP) é a parede inter septal, e (PP) é a parede posterior do ventrículo esquerdo e 1,047(mg/ $\mathrm{mm}^{3}$ ) corresponde a densidade do miocárdio.

\section{Índice de performance miocárdica}

O índice de performance miocárdica (IPM) foi calculado pela soma do tempo de contração isovolumétrico e o tempo de relaxamento isovolumétrico dividido pelo tempo de ejeção.

\section{Coleta do ventrículo esquerdo}

Vinte e quatro horas após a última sessão de treino, os animais foram decapitados. O VE coletado foi pesado e rapidamente congelado em nitrogênio líquido e mantido congelado em freezer a $-80^{\circ} \mathrm{C}$ até a realização das análises bioquímicas e moleculares. A hipertrofia cardíaca foi determinada pela massa do ventrículo esquerdo (parede livre e septo) corrigido pelo comprimento ta tíbia $(\mathrm{VE} / \mathrm{T})(\mathrm{mg} / \mathrm{mm})$.

\section{Análises moleculares}

Determinação da expressão do gene da $\alpha$ e $\beta$ miosina de cadeia pesada

A expressão dos genes das isoformas de MCP no ventrículo esquerdo (VE) foram determinadas pela técnica de reação da polimerase em cadeia em tempo real (RT-PCR) conforme descrito abaixo:

Extração do RNA total: todo o procedimento foi realizado com a utilização de luvas, materiais e soluçōes autoclavadas reservadas para RNA, pela técnica de CHOMCZYNSKI e SACCHI (1987). As amostras com aproximadamente $0,5 \mathrm{~g}$ foram homogeneizadas em $5 \mathrm{~mL}$ de TRIzol $^{\circledR}$ Reagent (Invitrogen) e a extração foi realizada conforme as instruções do fabricante. O RNA precipitado foi lavado com etanol $70 \%$ para eliminar resíduos de fenol e sal, e solubilizado em água tratada com dietilpirocarbonato (DEPC). A concentração das amostras de RNA total foi determinada por espectrofotometria no comprimento de onda de 260 $\mathrm{nm}$. A integridade da amostra foi verificada através de eletroforese em gel de agarose $1 \%$, contendo 0,5 $\mu \mathrm{g} / \mathrm{mL}$ de brometo de etídeo. O gel foi imerso em tampão TAE $1 X$ e a eletroforese realizada a 100 Volts por aproximadamente 20 minutos. A qualidade das amostras foi avaliada pela análise da intensidade das bandas correspondentes às subunidades do RNA ribossomal $28 \mathrm{~S}$ e $18 \mathrm{~S}$. Amostras que apresentaram algum grau de degradação foram descartadas.

Sintese de $c D N A$ : para a síntese de cDNA foram utilizados $2 \mu \mathrm{g}$ de RNA total, extraídos a partir do tecido cardíaco (VE) dos ratos. As amostras foram incubadas com $0,5 \mu \mathrm{g} / \mathrm{mL}$ de oligo dT12-18 a 65 ${ }^{\circ} \mathrm{C}$ por cinco minutos, para se obter a primeira fita de cDNA. A transcrição reversa das amostras foi realizada em um volume total de $20 \mu \mathrm{L}$ contendo $3 \mathrm{U}$ de RNAsin (Promega, Madison, USA), dNTPs $10 \mathrm{mM}$, DTT 0,1 M, tampão da enzima 1X, e 2,5U de SuperScript Reverse Transcriptase II (Invitrogen, 
Brasil). Após incubação por uma hora a $42^{\circ} \mathrm{C}$, a temperatura foi elevada a $95^{\circ} \mathrm{C}$ por cinco minutos e as amostras rapidamente colocadas em gelo para desnaturação de híbridos RNA-cDNA formados e inativação da enzima utilizada na reação. $O$ cDNA obtido foi estocado no freezer a $-20^{\circ} \mathrm{C}$ até a realização da reação de "Real Time" PCR.

Real Time PCR: foi realizado pelo sistema de detecção do produto específico amplificado no equipamento ABI 7700 (Applied-Biosystems), na presença do composto fluorescente SYBR-Green I. A otimização da reação de "Real Time" PCR foi feita conforme as instruções do fabricante (AppliedBiosystems, boletim do usuário no 2 , aplicado ao protocolo SYBR-Green I), corrigido para o volume final de $20 \mu \mathrm{l}$ por reação. As condições da reação foram padrão (protocolo do "kit" SYBR-Green I "master mix") e todos os reagentes foram fornecidos pelo "kit", inclusive a enzima polimerase AmpliTaq-Gold
(Applied-Biosystems). Depois da otimização, os "primers" foram utilizados na concentração de 200 nM para a detecção e a quantificação relativa da expressão dos genes da ciclofilina (gene controleinterno). A expressão dos genes das isoformas $\alpha$ e $\beta$ de MCP foram realizadas no ventrículo esquerdo dos ratos sedentários e treinados.

\section{Análise estatística}

Os dados estão representados como média \pm EPM. Para a comparação entre os grupos utilizamos análise de variância (ANOVA de duas entradas) sendo adotado a $\mathrm{MC}$ e o treinamento físico como fatores fixos. Quando encontrada significância no valor de $\mathrm{F}$ foi aplicado o teste de Bonferroni como "pos-hoc" para múltiplas comparações. Foi adotado para todos os experimentos um $\mathrm{p} \leq 0,05$ de significância.

\section{Resultados}

\section{Variáveis cardiorrespiratórias e hemodinâmicas}

\section{Consumo de oxigênio depico evelocidade decorrida}

Inicialmente, para mostrar que o protocolo de treinamento físico utilizado neste estudo foi efetivo, medimos o consumo de oxigênio de pico a velocidade máxima e o tempo de corrida dos animais no final do período do TF. Ambos os grupos obesos tiveram um menor consumo de oxigênio de pico, quando comparados com os grupos magros, entretanto, o grupo GOTR apresentou aumento de 21\% (GOTR $=54,13 ; \mathrm{GO}=44,88 \mathrm{ml} / \mathrm{kg} / \mathrm{min})(\mathrm{p}<0,05)$ do consumo de oxigênio de pico, quando comparado com o seu controle sedentário. Ainda, os grupos treinados atingiram tanto a velocidade (GOTR $=50 \%$; GMTR $=22 \%)$, quanto o tempo $(\mathrm{GOTR}=54 \%$; GMTR = $39 \%)(\mathrm{p}<0,05)$ de corrida maior quando comparados com os seus respectivos controles (FIGURA 1).

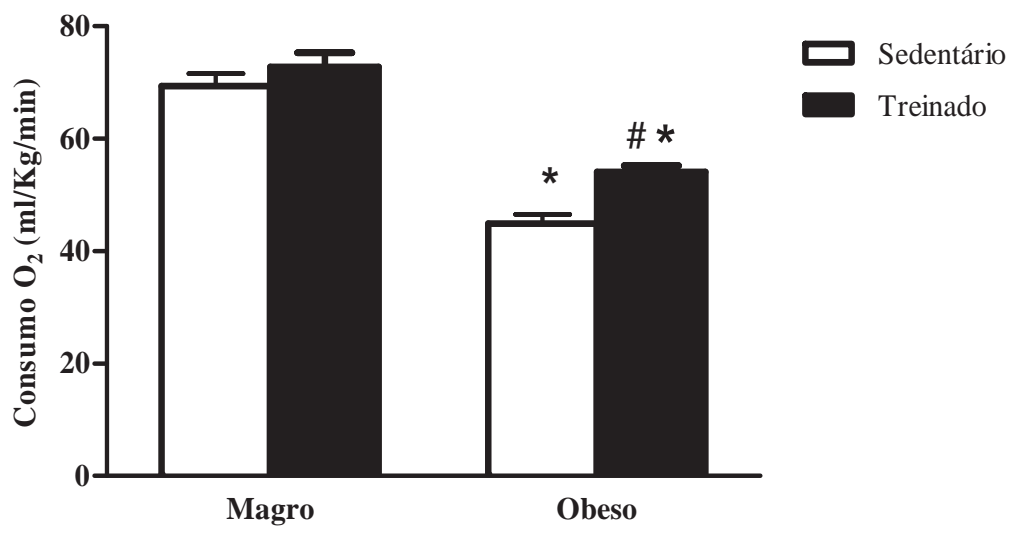

Os dados estão expressos como média \pm EPM para ratos do grupo magro (GM), grupo magro treinado (GMTR), grupo obeso (GO) e grupo obeso treinado (GOTR); * $p<0,05$ vs grupo magro; $\# p<0,05$ vs grupo obeso $(n=5 /$ grupo)

FIGURA 1 - Consumo de oxigênio de pico nos diferentes grupos. 
Os dados estão expressos como média \pm EPM para ratos do grupo magro (GM), grupo magro treinado (GMTR), grupo obeso (GO) e grupo obeso treinado (GOTR); ${ }^{*} p<0,05$ vs grupo magro; $\# p<0,05$ vs grupo obeso; ( $n=5 /$ grupo).

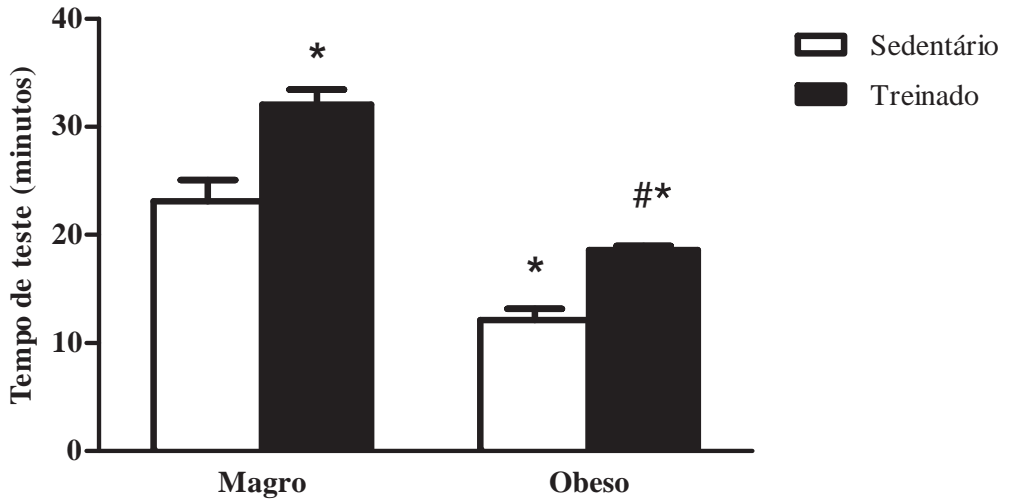

FIGURA 2 - Teste progressivo até a exaustão.

Frequência cardíaca

Outro dado que confirma a eficácia do TF é a bradicardia de repouso. A FC de repouso apresentou uma diminuição de aproximadamente $12 \%$ para ambos os grupos treinados, quando comparados com seus respectivos controles ( $\mathrm{p}<$ 0,05) (TABELA 1).

Além da FC também foi analisada a PAS. Conforme discutido acima, os grupos treinados apresentaram bradicardia de repouso, entretanto, a PAS aumentou em ambos os grupos obesos, conforme demonstrado na TABELA 1.
Os resultados estão expressos como média \pm EPM para ratos do grupo magro (GM), grupo magro treinado (GMTR), grupo obeso (GO) e grupo obeso treinado (GOTR); ${ }^{*} \mathrm{p}<0,05$ vs $\mathrm{GM}$; ${ }^{*} p<0,05$ vs GO; ( $n=5 /$ grupo).
Os resultados estão expressos como média \pm EPM para MCl (massa corporal inicial), MCF (massa corporal final), DM (delta ganho de massa), VE/T (ventrículo esquerdo/tíbia), GV (gordura visceral), para ratos do grupo magro (GM), grupo magro treinado (GMTR), grupo obeso (GO) e grupo obeso treinado (GOTR); *p $<0,05$ vs GM; $\# p<0,05$ vs GO; ( $n=5$ /grupo).

TABELA 1 - Efeitos da obesidade e do treinamento físico nas medidas hemodinâmicas.

\begin{tabular}{lcccc}
\hline Medidas hemodinâmicas & GM & GMTR & GO & GOTR \\
\hline FC $(\mathrm{bpm})$ & $437 \pm 15$ & $383 \pm 11^{*}$ & $433 \pm 21$ & $383 \pm 06^{\#}$ \\
PAS $(\mathrm{mmHg})$ & $115 \pm 02$ & $116 \pm 01$ & $124 \pm 02^{*}$ & $127 \pm 04^{*}$ \\
\hline
\end{tabular}

\section{Parâmetros corporais}

A TABELA 2 mostra os valores de MC inicial e final, do delta da massa, da massa do VE corrigido pelo comprimento da tíbia (T) (VE/T) e da gordura visceral (GV). Os grupos obesos apresentaram uma MC inicial significativamente maior $(\mathrm{GO}=45 \%$; GOTR $=52 \%)$, em relação ao grupo GM $(\mathrm{p}<0,01)$, porém, os grupos magros e obesos não foram diferentes entre si. O mesmo comportamento foi verificado em relação à $\mathrm{MC}$ final, $(\mathrm{GO}=59 \%$; GOTR = 53\%), em relação ao grupo GM ( $<<0,01)$. Entretanto, quando foram comparadas as médias do ganho de massa final menos a massa inicial (delta massa), o grupo GO teve um aumento de $107 \%$ da MC em relação ao grupo GM ( $\mathrm{p}<0,01)$, e $145 \%$ em relação ao grupo GOTR ( $\mathrm{p}<$ 0,01 ), enquanto que o grupo GOTR não foi diferente dos grupos GM e GMTR.

Em relação a razão $\mathrm{VE} / \mathrm{T}$, foi observado aumento significativo nos grupos GO e GOTR (GO = 37\%; GOTR $=19 \%$ ), quando comparado com o grupo GM $(\mathrm{p}<0,05)$. Por outro lado, o grupo GOTR teve uma redução de $13 \%$ na massa do $\mathrm{VE}$, quando comparado ao grupo $\mathrm{GO}(\mathrm{p}<0,05)$. Já a gordura visceral aumentou $750 \%$ no grupo GO e $580 \%$ no grupo GOTR comparado com o grupo GM ( $p<0,001)$, enquanto que o treinamento físico reduziu a GV em $20 \%$ no grupo GOTR, quando comparado ao grupo GO $(\mathrm{p}<0,05)$.

TABELA 2 -Efeitos da obesidade e do treinamento físico nas medidas corporais.

\begin{tabular}{lcccc}
\hline Medidas corporais & GM & GMTR & GO & GOTR \\
\hline MCI $(\mathrm{g})$ & $349,06 \pm 23,83$ & $342,64 \pm 27,58$ & $507,10 \pm 39,23^{*}$ & $532,95 \pm 33,88^{*}$ \\
MCF $(\mathrm{g})$ & $378,70 \pm 30,00$ & $383,40 \pm 27,93$ & $600,92 \pm 38,28^{*}$ & $580,75 \pm 44,54^{*}$ \\
DP $(\mathrm{g})$ & $45,65 \pm 12,10$ & $40,76 \pm 10,17$ & $93,82 \pm 29,05$ & $48,14 \pm 24,44^{\#}$ \\
Razão VE/T $(\mathrm{mg} / \mathrm{mm})$ & $1,94 \pm 0,12$ & $1,80 \pm 0,09$ & $2,65 \pm 0,12^{*}$ & $2,31 \pm 0,10^{* \#}$ \\
GV $(\mathrm{g})$ & $10,76 \pm 0,97$ & $9,92 \pm 1,30$ & $85,00 \pm 6,06^{*}$ & $68,25 \pm 2,36^{* \#}$ \\
\hline
\end{tabular}

598 • Rev. bras. Educ. Fís. Esporte, São Paulo, v.25, n.4, p.593-605, out./dez. 2011 


\section{Marcadores de hipertrofia cardíaca patológica}

Para constatar se o aumento da massa do VE estava relacionado com uma HC patológica, analisamos marcadores gênicos de HC patológica pela técnica
Real Time PCR. Conforme demonstrado na FIGURA 3a, b e c, a obesidade não alterou a expressão da $\alpha M C P$, enquanto que aumentou a expressão da BMCP $(p<0,05)$, desta forma resultando em aumento da relação $\alpha / \beta \mathrm{MCP}(\mathrm{p}<0,05)$, enquanto que o TF aeróbio reverteu parcialmente essa alteração.

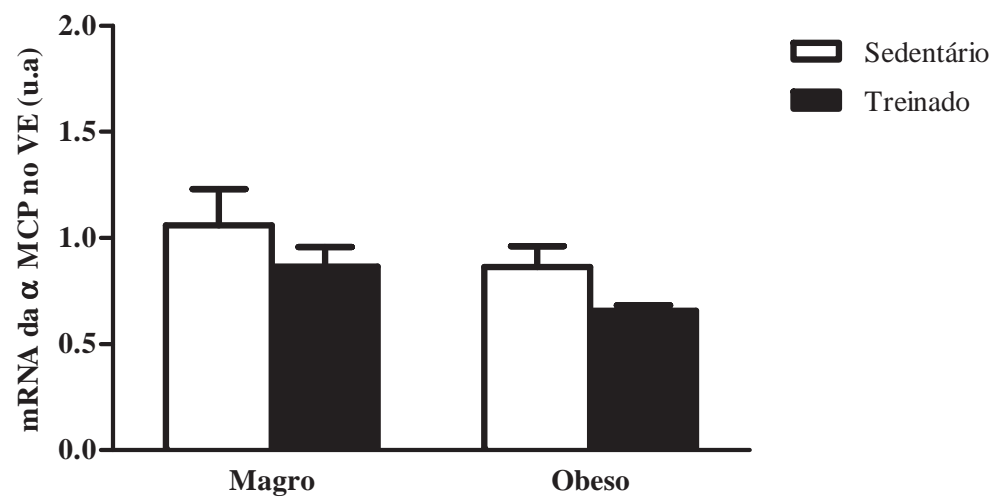

Os dados estão expressos como média \pm EPM para ratos do grupo magro (GM), grupo magro treinado (GMTR), grupo obeso (GO) e grupo obeso treinado (GOTR).

FIGURA 3a - Expressão gênica do mRNA da a miosina de cadeia pesada ( $\alpha \mathrm{MCP})$ no ventrículo esquerdo.

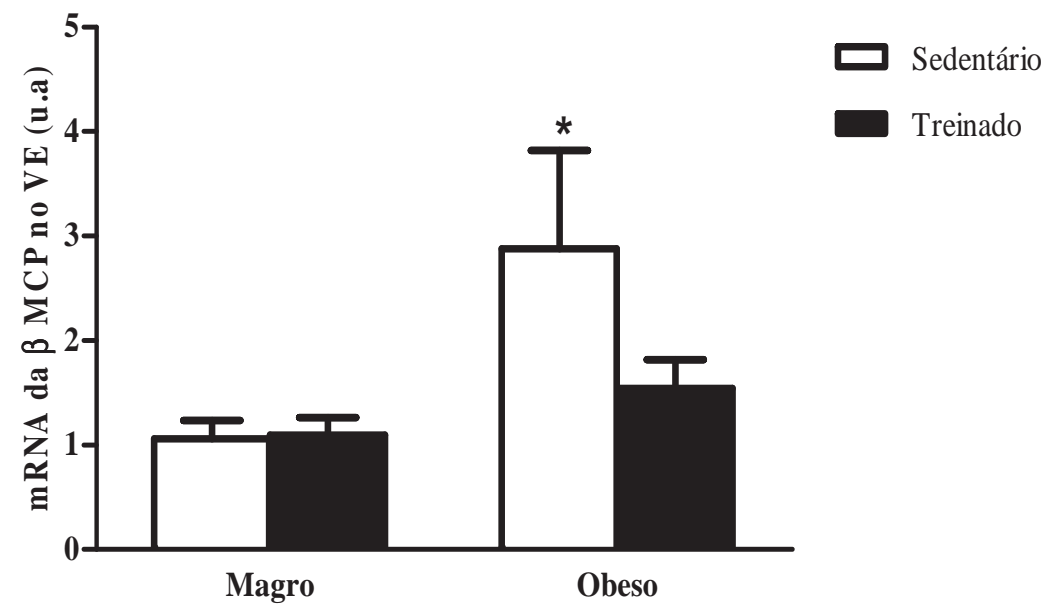

Os dados estão expressos como média \pm EPM para ratos do grupo magro (GM), grupo magro treinado (GMTR), grupo obeso (GO) e grupo obeso treinado (GOTR); ${ }^{*} p<0,05$ vs grupo magro.

Os dados estão expressos como média \pm EPM para ratos do grupo magro (GM), grupo magro treinado (GMTR), grupo obeso (GO) e grupo obeso treinado (GOTR). ${ }^{*} \mathrm{p}<$ vs grupo magro. 


\section{Função cardíaca}

As alterações cardíacas morfológicas (massa do $\mathrm{VE}$ ) e moleculares (expressão gênica da MCP), observadas, podem levar a alteraçôes funcionais cardíacas. Assim, analisamos a função cardíaca através do ecocardiograma.

Os dados apresentados na TABELA 3 mostram as análises da função sistólica, pela análise da fração de ejeção e de encurtamento do VE. Nenhum dos grupos apresentou diferenças para ambas as medidas, o que mostra que a obesidade não resultou em prejuízos da função sistólica. Entretanto, quando avaliada a função diastólica, pelo tempo de relaxamento isovolumétrico e pela relação do pico da onda (E) pelo pico da onda (A), (relação
E/A), o grupo GO apresentou uma redução (-36\%) dessa relação, quando comparado com o grupo GM ( $\mathrm{p}<0,05)$, o que indica um prejuízo de função diastólica. Ainda, foi verificado que o grupo GOTR reverteu esse prejuízo, não sendo mais diferente dos grupos GM, GMTR. Neste estudo, também foi observado aumento da massa do VE no grupo GO $(+26 \%)(p<0,05)$, esses dados corroboram o resultado obtido pela análise da razão do $\mathrm{VE} / \mathrm{T}$ $(+37 \%)(p<0,05)$, confirmando a HC nesse modelo de obesidade. Outro resultado interessante obtido pelo ecocardiograma foi o aumento do IPM em ambos os grupos treinados $(\mathrm{p}<0,05)$, o que também corrobora com os resultados de bradicardia e consumo de oxigênio, como mais um marcador de treinamento físico.
Os resultados estão expressos como média \pm EPM para Fej (fração de ejeção do ventrículo esquerdo), Fen (fração de encurtamento do ventrículo esquerdo), TRIV (tempo de relaxamento isovolumétrico), MVE (massa do ventrículo esquerdo), IPM (índice de performance miocárdica), para ratos do grupo magro (GM), grupo magro treinado (GMTR), grupo obeso (GO) e grupo obeso treinado (GOTR);

${ }^{*} \mathrm{p}<0,05$ vs $\mathrm{GM}$;

" $p<0,05$ vs $G 0$;

( $n=5 /$ grupo).

TABELA 3 -Efeitos da obesidade e do treinamento físico na função cardíaca.

\begin{tabular}{lcccc}
\hline Função cardíaca & GM & GMTR & GO & GOTR \\
\hline Função sistólica & & & & \\
Fej (\%) & $0,83 \pm 0,03$ & $0,78 \pm 0,08$ & $0,88 \pm 0,02$ & $0,84 \pm 0,03$ \\
Fen (\%) & $0,45 \pm 0,02$ & $0,42 \pm 0,08$ & $0,52 \pm 0,03$ & $0,48 \pm 0,03$ \\
& & & & \\
Função diastólica & & & & \\
Relação E/A (m/s) & $2,40 \pm 0,11$ & $2,14 \pm 0,26$ & $1,54 \pm 0,26^{*}$ & $2,20 \pm 0,62^{*}$ \\
TRIV (ms) & $22,00 \pm 2,45$ & $29,40 \pm 9,13$ & $28,00 \pm 4,64$ & $29,50 \pm 3,46$ \\
& & & & \\
MVE (g) & $0,98 \pm 0,04$ & $1,17 \pm 0,03^{*}$ & $1,24 \pm 0,07^{*}$ & $1,02 \pm 0,12^{*}$ \\
IPM & $0,32 \pm 0,01$ & $0,45 \pm 0,03^{*}$ & $0,39 \pm 0,01$ & $0,49 \pm 0,02^{*}$ \\
\hline
\end{tabular}

\section{Discussão}

Este estudo mostra que o TF aeróbio de natação promoveu alterações estruturais e moleculares cardíacas, as quais podem ter contribuído na melhora da função ventricular observada nos ratos Zucker obesos treinados.

Os ratos obesos apresentaram aumento da massa do VE acompanhada de alterações moleculares cardíacas e prejuízo na função diastólica. A associação do TF aeróbio a obesidade reverteu essas alterações cardíacas causadas pela obesidade e restabeleceu a função ventricular. Esses resultados demonstram pela primeira vez os efeitos do TF aeróbio na melhora dos parâmetros moleculares juntamente com a função cardíaca de ratos Zucker obesos. Nesse sentido, o TF aeróbio é uma importante forma de tratamento nãofarmacológico para as doenças cardíacas decorrentes do sobrepeso ou da obesidade.

Inicialmente, para confirmar a eficácia do protocolo de treinamento avaliamos dois marcadores de TF: a FC de repouso e o consumo de oxigênio de pico no exercício. A bradicardia de repouso consiste em um bom parâmetro funcional para verificar as adaptações e eficácia do TF aeróbio, sendo, portanto, considerada um marcador fisiológico de TF aeróbio (De Angelis, Oliveira, Werner, Bock, Bello-Klein, Fernandes, Bello \& Irigoyen, 1997; Trapp, Chisholm, Freund \& Boutcher, 2008). Entretanto, a magnitude da bradicardia de 
repouso é dependente do tipo, intensidade e espécie submetida ao TF aeróbio. Estudos mostram uma maior bradicardia de repouso em animais treinados com natação (Medeiros et al., 2004), do que em esteira rolante (NEgrāo, Moreira, SAntos, Farah \& Krieger, 1992). Estas diferenças podem ser dependentes dos diferentes mecanismos que levam a diminuição da FC no repouso.

No presente estudo a $\mathrm{FC}$ de repouso foi menor nos grupos treinados com natação, quando comparado com seus respectivos controles. Embora não tenhamos investigado o mecanismo de redução da FC neste este estudo, Medeiros et al. (2004) observaram que a bradicardia de repouso em ratos Wistar treinados com natação ocorreu devido ao aumento do tônus vagal. Em ratos normotensos, a bradicardia de repouso resultante do $\mathrm{TF}$ aeróbio em esteira está associada a redução na FC intrínseca (Evangelista, Martuchi, Negrão \& Brum, 2005; NEGRÃO et al., 1992). Enquanto, em ratos SHR, um modelo experimental de hipertensão espontânea, a diminuição da FC de repouso no animal treinado parece ocorrer devido a redução do tônus simpático cardíaco (Gava, Veras-Silva, Negrāo \& Krieger, 1995), o que sugere que diferentes mecanismo podem estar envolvidos na resposta bradicárdica com o TF.

Outros marcadores de TF utilizados foram o consumo de oxigênio de pico, a velocidade e o tempo final atingido em esteira. $\mathrm{O}$ aumento do consumo de oxigênio de pico com o TF aeróbio ocorre devido a fatores, como: aumento da capilarização, aumento da diferença arteriovenosa e aumento do débito cardíaco durante o exercício (Trapp et al., 2008). O grupo GOTR apresentou aumento significativo tanto do consumo de oxigênio de pico quanto na velocidade e tempo de corrida quando comparado com seu respectivo controle. Já para o grupo GMTR não foram apresentadas alterações no consumo de oxigênio de pico, contudo, observamos maior tolerância ao exercício como demonstrado pelo maior tempo e distância final de corrida.

Um dos fatores que podem ter prejudicado o aumento no consumo de oxigênio de pico do grupo GMTR é a falta de especificidade do teste, haja vista que o protocolo de treinamento foi de natação. Outro fator que deve ser levado em consideração é a MC dos animais, pois, sabe-se que em valores absolutos, a obesidade gera aumento do consumo de oxigênio, tanto em repouso, quanto durante a realização do exercício, pois, obesos possuem uma maior demanda metabólica em ambas as situações (SoOD, 2009), o que poderia explicar o aumento do consumo de oxigênio de pico mais acentuado no grupo GOTR, uma vez que o exercício em esteira possa representar uma maior sobrecarga para o obeso. Enquanto que no protocolo de natação a sobrecarga foi ajustada de acordo com a MC de cada animal. Dessa forma, embora não encontrado aumento no consumo de oxigênio de pico para o grupo GOTR, os dados de FC e tolerância ao exercício demonstram que o protocolo de treinamento físico aeróbio de natação adotado foi eficaz em produzir uma melhora tanto na condição cardiovascular quanto nos parâmetros de condicionamento físico para ambos os grupos treinados.

O aumento da massa e gordura corporal levam ao sobrepeso e são excelentes parâmetros para determinar o desenvolvimento da obesidade. Por outro lado, o exercício físico aeróbio é um coadjuvante no controle de ganho de massa e gordura corporal (OHKAWARA, TANAKA, MiYaChI, ISHIKAWA-TANAKA \& TABATA, 2007), pois promove aumento no consumo energético, em parte pelo aumento da atividade simpática para o tecido adiposo branco (BULow, 2004), desta forma aumentando o gasto calórico e diminuindo a gordura corporal (BuLow, 2004).

O exercício também atua na prevenção da perda de massa magra (Kempen, Saris \& Westerterp, 1995), que é um importante efeito, pois previne a queda do metabolismo de repouso aumentando a perda da MC. Entretanto, dados da literatura mostram que o exercício físico aeróbio por si só não possui efeito pronunciado na perda da $\mathrm{MC}$, sendo necessário a associação de dieta alimentar (Skender, GoOdrick, Del Junco, ReEves, Darnell, Gotto \& Foreyt, 1996; Trombetta, Batalha, Rondon, Laterza, Kuniyoshi, Gowdak, Barretto, Halpern, Villares \& Negrāo, 2003). Walberg, Mole e STERn (1982) mostraram que o TF de natação na ausência de dieta, apenas previne o ganho acentuado da massa e da gordura corporal, enquanto que a associação com a dieta foi mais eficiente na redução desses parâmetros em ratos Zucker obesos. No presente estudo não foi controlada a dieta alimentar, o que pode ter contribuído para a manutenção da MC no final do protocolo experimental em comparação ao início nos animais treinados, embora o grupo obeso treinado (GOTR) tenha ganhado aproximadamente $50 \%$ menos $\mathrm{MC}$, quando comparado ao grupo sedentário. Desta forma, semelhante aos dados de WalBerg, Mole e STERN (1982), observamos que o TF foi eficaz em prevenir o ganho acentuado da MC e da GV. Essa prevenção pode ser benéfica, uma vez que o aumento tanto da MC quanto da GV são fatores de risco à saúde. 
Neste estudo, observamos que o aumento da MC e da GV foram acompanhados por aumento da massa do VE analisados tanto pela razão VE/T, quanto pelo ecocardiograma. A obesidade está relacionada a alterações cardíacas tanto em humanos (ABEL, LITWIN \& SWEENEY, 2008), quanto em modelos de animais (ABEL, LITWIN \& SWEENEY, 2008). Dentre as principais alteraçōes temos o aumento da massa ventricular, da câmara atrial, prejuízos sub-clínicos das funções diastólica e sistólica (ABEL, LITWIN \& SWEENEY, 2008). A HC tem sido observada na maioria dos modelos animais de obesidade, entretanto, parecem existir algumas diferenças em relação à idade, tempo de dieta e espécie, como demonstrado por alguns autores (Carroll, Zenebe \& Strange, 2006; OuWens, Boer, Fodor, de Galan, Heine, MaAsen \& Diamant, 2005). Ratos Zucker obesos apresentam aumento da massa cardíaca, quando comparado com o seu controle magro (Duarte, Martinez, Bermejo, Vera, Gamez, Cabo \& ZarZuelo, 1999). O aumento da massa cardíaca em modelos experimentais de obesidade tem sido relacionado a fatores como: aumento da demanda metabólica periférica (que por sua vez causa um maior estresse cardíaco, tanto na pré, quanto na póscarga), maior acúmulo de lipídios cardíaco (pois, obesos possuem alterações metabólicas cardíacas) e aumento do colágeno cardíaco (ChristofFersen, Bollano, Lindegaard, Bartels, Goetze, Andersen \& Nielsen, 2003; Semeniuk, Kryski \& Severson, 2002). Esses efeitos quando sustentados por longo prazo podem ocasionar disfunção diastólica, como encontrada no presente estudo.

A disfunção diastólica é bem demonstrada em indivíduos obesos. Ratos Zucker obesos apresentam aumento de fibrose cardíaca (ToBLLI et al., 2005, 2006) e da expressão da $\beta \mathrm{MCP}$, sem alterações da $\alpha \mathrm{MCP}$ (GolfMAN et al., 2005). Estas alteraçôes determinam modificaçôes na capacidade contrátil do miocárdio, o que pode levar à diminuição na velocidade de encurtamento dos sarcômeros e causar disfunção cardíaca (IzUMO et al., 1988; SWYNGHEDAuw, 2006). Dessa forma, com o intuito de melhor entender o prejuízo diastólico e observar se o TF era capaz de reverter a disfunção cardíaca, analisamos a expressão gênica de duas importantes proteínas contráteis ( $\alpha$ e $\beta$-MCP). Assim, demonstramos que a obesidade levou a diminuição da relação $\alpha / \beta \mathrm{MCP}$, e essa alteração foi devido ao aumento da expressão da BMCP, a qual apresenta baixa atividade da ATPase miosínica e pode levar a prejuízo na função contrátil do miocárdio. Por outro lado, o TF foi capaz de reverte esse efeito aumentando a relação $\alpha / \beta \mathrm{MCP}$, assim como prevenindo o prejuízo de função diastólica. A diminuição da $\beta M C P$ no grupo GOTR pode contribuir para a melhora na função diastólica, uma vez que maiores quantidades de $\beta \mathrm{MCP}$ geram diminuição na velocidade de encurtamento dos sarcômeros e perda na função contrátil. Resultados semelhantes foram observados em SHR, onde ratos treinados com natação apresentaram aumento na atividade da ATPase miosínica no ventrículo esquerdo, o qual foi secundário ao aumento da expressão da isoforma $\alpha$-MCP, melhorando a função sistólica do animal treinado (SCHAible \& SCHEuer, 1981).

Em conclusão, os resultados em conjunto sugerem que os animais geneticamente obesos apresentam na idade adulta um fenótipo cardíaco patológico, o qual foi revertido pelo TF. Portanto, o TF aeróbio de natação foi capaz de prover alterações moleculares e estruturais cardíacas prevenindo a disfunção ventricular em ratos Zucker obesos. Desta forma, o estudo também mostra que estes animais são um bom modelo para investigação da fisiopatologia da obesidade.

\begin{abstract}
Aerobic exercise training revert pathologic cardiac hypertrophy and improves the diastolic function in obese Zucker rats

Obesity is profoundly involved in cardiovascular diseases. On the other hand, aerobic exercise training (EXT) attenuates obesity and promotes cardiac benefits in obese individuals. Therefore, the aim of this study was to investigate if obesity alters the cardiac function and whether its association with exercise training can improve cardiac function in an obese Zucker rat strain. The rats were divided in the following groups: Lean Zucker rats (LZR); lean Zucker rats plus exercise training (LZR+EXT); obese Zucker rat (OZR) and obese Zucker rat plus exercise training (OZR+EXT). EXT consisted of 10 weeks swimming sessions of $60 \mathrm{~min}, 5$ days/week. At the end of the training protocol we evaluated heart rate (HR), systolic blood
\end{abstract}


pressure (SBP), cardiac hypertrophy $(\mathrm{CH})$ and function. The trained groups $\mathrm{LZR}+\mathrm{EXT}$ and OZR+EXT showed a 12\% lower resting HR when compared with theirs respective controls. In addition, our results showed that exercise training reduced the cardiac mass by $13 \%$ and improved the diastolic function by $43 \%$ in the obese trained group when compared with the obese untrained. In conclusion, aerobic exercise training reverts the cardiac injuries in obese Zucker rats.

UnIterms: Obesity; Cardiac disease; Aerobic exercise training; Zucker rats.

\section{Referências}

ABEL, E.D.; LITWIN, S.E.; SWEENEY, G. Cardiac remodeling in obesity. Physiological Reviews, Bethesda, v.88, n.2, p.389-419, 2008.

BERNSTEIN, D. Exercise assessment of transgenic models of human cardiovascular disease. Physiological Genomics, Bethesda, v.13, n.3, p.217-26, 2003.

BRENNER, D.A.; APSTEIN, C.S.; SAUPE, K.W. Exercise training attenuates age-associated diastolic dysfunction in rats. Circulation, Hagerstown, v.104, n.2, p.221-6, 2001.

BROOKS, G.A.; WHITE, P. Determination of metabolic and heart rate responses of rats to treadmill exercise. Journal of Applied Physiology, Bethesda, v.45, n.6, p.1009-15, 1978.

BULOW, J. Physical activity and adipose tissue metabolism. Scandinavian Journal of Medicine and Science in Sports, Copenhagen, v.14, n.2, p.72-3, 2004.

CARROLL, J.F.; ZENEBE, W.J.; STRANGE, T.B. Cardiovascular function in a rat model of diet-induced obesity. Hypertension, Dallas, v.48, n.1, p.65-72, 2006.

CHOMCZYNSKI, P.; SACCHI, N. Single-step method of RNA isolation by acid guanidinium thiocyanate-phenolchloroform extraction. Analytical Biochemistry, Orlando, v.162, n.1, p.156-9, 1987.

CHRISTOFFERSEN, C.; BOLLANO, E.; LINDEGAARD, M.L.; BARTELS, E.D.; GOETZE, J.P.; ANDERSEN, C.B.; NIELSEN, L.B. Cardiac lipid accumulation associated with diastolic dysfunction in obese mice. Endocrinology, Chevechase, v.144, n.8, p.3483-90, 2003.

COLÉGIO BRASILEIRO DE EXPERIMENTAÇÃO ANIMAL (COBEA). Princípios éticos na experimentação animal. Brasília: COBEA, 1991. Disponível em: <www.cobea.org.br>.

De ANGELIS, K.L.; OLIVEIRA, A.R.; WERNER, A.; BOCK, P.; BELLO-KLEIN, A.; FERNANDES, T.G.; BELLO, A.;A.; IRIGOYEN, M.C. Exercise training in aging: hemodynamic, metabolic, and oxidative stress evaluations. Hypertension, Dallas, v.30, n.3, Pt 2, p.767-71, 1997.

DEITEL, M. Overweight and obesity worldwide now estimated to involve 1.7 billion people. Obesity Surgery, New York, v.13, n.3, p.329-30, 2003.

DUARTE, J.; MARTINEZ, A.; BERMEJO, A.; VERA, B.; GAMEZ, M.J.; CABO, P.; ZARZUELO, A. Cardiovascular effects of captopril and enalapril in obese Zucker rats. European Journal of Pharmacology, Amsterdam, v.365, n.2-3, p.225-32, 1999. EVANGELISTA, F.S.; MARTUCHI, S.E.; NEGRAO, C.E.; BRUM, P.C. Loss of resting bradycardia with detraining is associated with intrinsic heart rate changes. Brazilian Journal of Medical and Biological Research, Ribeirão Preto, v.38, n.7, p.1141-6, 2005.

GAVA, N.S.; VERAS-SILVA, A.S.; NEGRAO, C.E.; KRIEGER, E.M. Low-intensity exercise training attenuates cardiac betaadrenergic tone during exercise in spontaneously hypertensive rats. Hypertension, Dallas, v.26, n.6, Pt 2, p.1129-33, 1995. GOLFMAN, L.S.; WILSON, C.R.; SHARMA, S.; BURGMAIER, M.; YOUNG, M.E.; GUTHRIE, P.H.; VAN ARSDALL, M.; ADROGUE, J.V.; BROWN, K.K.; TAEGTMEYER, H. Activation of PPARgamma enhances myocardial glucose oxidation and improves contractile function in isolated working hearts of ZDF rats. American Journal of Physiology, Endocrinology and Metabolism, Bethesda, v.289, n.2, p.E328-36, 2005.

GUPTA, M.P. Factors controlling cardiac myosin-isoform shift during hypertrophy and heart failure. Journal of Molecular, Cellular and Cardiology, London, v.43, n.4, p.388-403, 2007.

IZUMO, S.; NADAL-GINARD, B.; MAHDAVI, V. Protooncogene induction and reprogramming of cardiac gene expression produced by pressure overload. Proceedings of the National Academy of Sciences of the United States of America, Washington, v.85, n.2, p.339-43, 1988. 
JIN, H.; YANG, R.; LI, W.; LU, H.; RYAN, A.M.; OGASAWARA, A.K.; VAN PEBORGH, J.; PAONI, N.F. Effects of exercise training on cardiac function, gene expression, and apoptosis in rats. American Journal of Physiology, Heart and Circulatory Physiology, Bethesda, v.279, n.6, p.H2994-3002, 2000.

KEMPEN, K.P.; SARIS, W.H.; WESTERTERP, K.R. Energy balance during an 8-wk energy-restricted diet with and without exercise in obese women. American Journal of Clinical Nutrition, Bethesda, v.62, n.4, p.722-9, 1995.

KENCHAIAH, S.; EVANS, J.C.; LEVY, D.; WILSON, P.W.; BENJAMIN, E.J.; LARSON, M.G.; KANNEL, W.B.; VASAN, R.S. Obesity and the risk of heart failure. New England Journal of Medicine, Boston, v.347, n.5, p.305-13, 2002. LITWIN, S.E.; KATZ, S.E.; MORGAN, J.P.; DOUGLAS, P.S. Serial echocardiographic assessment of left ventricular geometry and function after large myocardial infarction in the rat. Circulation, Hagerstown, v.89, n.1, p.345-54, 1994. MEDEIROS, A.; OLIVEIRA, E.M.; GIANOLLA, R.; CASARINI, D.E.; NEGRAO, C.;E.; BRUM, P. Swimming training increases cardiac vagal activity and induces cardiac hypertrophy in rats. Brazilian Journal of Medical and Biological Research, Ribeirão Preto, v.37, n.12, p.1909-17, 2004.

NEGRAO, C.E.; MOREIRA, E.D.; SANTOS, M.C.; FARAH, V.M.; KRIEGER, E.M. Vagal function impairment after exercise training. Journal of Applied Physiology, Bethesda, v.72, n.5, p.1749-53, 1992.

O’ROURKE, R.A.; HANRATH, P.; HENRY, W.N.; HUGENHOLTZ, P.G.; PISA, Z.; ROELANDT, J.; TANAKA, M. [Report of the Joint Committee of the International Society and Federation of Cardiology and the World Health Organization on the recommendation for the standardization of quantitation of M-mode echocardiography]. Archivos del Instituto de Cardiologia de Mexico, Mexico, v.54, n.4, p.405-9, 1984.

OHKAWARA, K.; TANAKA, S.; MIYACHI, M.; ISHIKAWA-TAKATA, K.; TABATA, I. A dose-response relation between aerobic exercise and visceral fat reduction: systematic review of clinical trials. International Journal of Obesity, London, v.31, n.12, p.1786-97, 2007.

OUWENS, D.M.; BOER, C.; FODOR, M.; DE GALAN, P.; HEINE, R.J.; MAASSEN, J.A.; DIAMANT, M. Cardiac dysfunction induced by high-fat diet is associated with altered myocardial insulin signalling in rats. Diabetologia, Berlin, v.48, n.6, p.1229-37, 2005.

POIRIER, P.; GILES, T.D.; BRAY, G.A.; HONG, Y.; STERN, J.S.; PI-SUNYER, F.X.; ECKEL, R.H. Obesity and cardiovascular disease: pathophysiology, evaluation, and effect of weight loss: an update of the 1997 American Heart Association Scientific Statement on Obesity and Heart Disease from the Obesity Committee of the Council on Nutrition, Physical Activity, and Metabolism. Circulation, Hagerstown, v.113, n.6, p.898-918, 2006.

SCHAIBLE, T.F.; SCHEUER, J. Cardiac function in hypertrophied hearts from chronically exercised female rats. Journal of Applied Physiology, Bethesda, v.50, n.6, p.1140-5, 1981.

SCHILLER, N.B.; SHAH, P.M.; CRAWFORD, M.; DeMARIA, A.; DEVEREUX, R.; FEIGENBAUM, H.; GUTGESELL, H.; REICHEK, N.; SAHN, D.; SCHNITTGER, I. Recommendations for quantitation of the left ventricle by two-dimensional echocardiography. American Society of Echocardiography Committee on Standards, Subcommittee on Quantitation of Two-Dimensional Echocardiograms. Journal of the American Society of Echocardiography, St. Louis, v.2, n.5, p.358-67, 1989

SEMENIUK, L.M.; KRYSKI, A.J.; SEVERSON, D.L. Echocardiographic assessment of cardiac function in diabetic db/db and transgenic db/db-hGLUT4 mice. American Journal of Physiology, Heart and Circulatory Physiology, Bethesda, v.283, n.3, p.H976-82, 2002.

SKENDER, M.L.; GOODRICK, G.K.; DEL JUNCO, D.J.; REEVES, R.S.; DARNELL, L.; GOTTO, A.M.; FOREYT, J.P. Comparison of 2-year weight loss trends in behavioral treatments of obesity: diet, exercise, and combination interventions. Journal of American Dietetic Association, Chicago, v.96, n.4, p.342-6, 1996.

SOOD, A. Altered resting and exercise respiratory physiology in obesity. Clinics in Chest Medicine, Philadelphia, v.30, n.3, p.445-54, 2009.

SWYNGHEDAUW, B. Phenotypic plasticity of adult myocardium: molecular mechanisms. Journal of Experimental Biology, London, v.209, n.Pt 12, p.2320-7, 2006.

TOBLLI, J.E.; CAO, G.; DeROSA, G.; FORCADA, P. Reduced cardiac expression of plasminogen activator inhibitor 1 and transforming growth factor beta1 in obese Zucker rats by perindopril. Heart, London, v.91, n.1, p.80-6, 2005.

TOBLLI, J.E.; CAO, G.; RIVAS, C.; DeROSA, G.; DOMECQ, P. Angiotensin-converting enzyme inhibition reduces lipid deposits in myocardium and improves left ventricular function of obese zucker rats. Obesity, Silver Spring, v.14, n.9, p.1586-95, 2006.

TRAPP, E.G.; CHISHOLM, D.J.; FREUND, J.; BOUTCHER, S.H. The effects of high-intensity intermittent exercise training on fat loss and fasting insulin levels of young women. International Journal of Obesity, London, v.32, n.4, p.684-91, 2008.

604 • Rev. bras. Educ. Fís. Esporte, São Paulo, v.25, n.4, p.593-605, out./dez. 2011 
TROMBETTA, I.C.; BATALHA, L.T.; RONDON, M.U.; LATERZA, M.C.; KUNIYOSHI, F.H.; GOWDAK, M.M.; BARRETTO, A.C.; HALPERN, A.; VILLARES, S.M.; NEGRAO, C.E. Weight loss improves neurovascular and muscle metaboreflex control in obesity. American Journal of Physiology, Heart and Circulatory Physiology, Bethesda, v.285, n.3, p.H974-82, 2003.

WALBERG, J.L.; MOLE, P.A.; STERN, J.S. Effect of swim training on development of obesity in the genetically obese rat. American Journal of Physiology, Bethesda, v.242, n.3, p.R204-11, 1982.

ENDEREÇO

Edilamar Menezes de Oliveira Lab. de Bioquímica da Atividade Motora Escola de Educação Física e Esporte - USP Av. Prof. Mello Moraes, 65 05508-030 - São Paulo - SP - BRASIL e-mail: edilamar@usp.br
Recebido para publicação: 20/01/2011

Revisado: 03/10/2011

Aceito: 04/10/2011 